\title{
The Numerical Simulation Analysis of Flow Field in Level Control Valve of Water Storage Tank
}

\author{
Yaping Wang ${ }^{1, \mathrm{a}}$, Lei Gao ${ }^{1, \mathrm{~b}}$, Jianghua $\mathrm{Ge}^{1, \mathrm{c}} \mathrm{Han} \mathrm{Gao}^{1 \mathrm{~d}}$ and Jing Zhang ${ }^{\text {le }}$ \\ ${ }^{1}$ School of Mechanical and Dynamic Engineering, Harbin University of Science and \\ Technology \\ awypbl@163.com, ${ }^{b}$ glsunyanzi@163.com, ${ }^{\mathrm{c}}$ gejianghua@sina.com, \\ dgaohan815@126.com, e21czhangjing@163.com
}

\begin{abstract}
Level control valve of water storage tank is installed in the outlet of tank, which control the water level of water storage tank by adjusting the water emissions, which make great significance for the safe operation of the super (super) criticality thermal power unit boiler. Complete the simulation analysis of internal flow field by the method of numerical simulation , in order to achieve structure improvements of level control valve of water storage tank, that make level control valve of water storage tank better meet the work requirements. Establish flow channel model and mesh models of level control valve of water storage tank in various stages of the open process. Make three dimensional viscous numerical simulation of the internal complex flow field by the computational fluid dynamics software of ANSYS CFX. The error between the simulation values of the flow coefficient and the theoretical values, trial values is less than $5 \%$, which verifies the correctness of the simulation results . Get pressure contour, velocity streamline diagrams and outlet mass flow of level control valve of water storage tank in various stages of the open process. According to the pressure contours and velocity streamline diagrams, make detailed analysis of the flow field of level control valve of water storage tank. According to the dates of outlet mass flow, fit the flow characteristic curve of level control valve of water storage tank. Provide a theoretical basis for the structural design of level control valve of water storage tank.
\end{abstract}

Keywords: Level control valve of water storage tank, Velocity streamline diagram, Pressure contour, Discharge coefficient, Curve of ideal flow characteristic

\section{Introduction}

As the rapid development of electric utilities in our country, supercritical technology has been widely used. The parameters, performance and yield of thermal power units has been occupies the first in the world. However, the manufacturing industry of valve has not kept pace with the development of power station equipment, and high-end valve of power station has been depending on imports, which has impacted on construction of power station in our country, and increase the cost of electric power construction greatly [1].

The ultra-supercritical boilers are important energy conversion devices of ultra supercritical units. Through the burning of coal, the boilers convert the chemical energy in coal into heat energy, pass the heat to the working fluid(water) which turns into steam with the ultra-supercritical parameters, then supply to the steam turbine. The circle system of ultra-supercritical boilers consists of built-in startup separator, water storage tank, recycle pump, down-flow tube, water connecting pipe, and riser-pipe of 
water cooling wall. When the working medium flowing from the outlet of water cooling wall is two-phase flow of steam and water, the separator plays a role of steam-water separation, pass the separating steam to the turbine. The boiler provides stable working conditions for separator. When water level of boiler is too high, the normal work of the separator will be damaged, if worse, the steam will take too much water, which will lead the increase of scaling on the turbine tube wall and affects quality of steam. The water of storage tank supply for recycling of boiler and protect the water cooling wall. When water level of boiler is too low, the water cycle will be damaged and causes a rupture of pipe of water cooling wall, if worse, pan will burns dry, water storage tank will be damaged [2]. Level control valve of water storage tank is installed in the outlet of tank, which control the water level of water storage tank by adjusting the water emissions of water storage tank. Complete the simulation analysis of internal flow field by the method of numerical simulation, which make great significance for the safe operation of the super (super) criticality thermal power unit boiler.

This paper complete the simulation analysis of internal flow field by the method of numerical simulation. Establish flow channel model and mesh models of level control valve of water storage tank in various stages of the open process. Get pressure contours, velocity streamline diagrams, outlet mass flow and discharge coefficient of level control valve of water storage tank in various stages of the open process. Make detailed analysis of the flow field of level control valve of water storage tank, and fit the flow characteristic curve of level control valve of water storage tank. Provide a theoretical basis for the structural design of level control valve of water storage tank.

\section{Governing Equations}

Assume that the Flow field inside the valve is three-dimensional incompressible viscous fluid [3].Governing equations of incompressible viscous fluid without heat exchange consists of mass-conservation equations (continuity equations) and momentum-conservation equations(N-S equations) [4].

$$
\begin{aligned}
& \frac{\partial u}{\partial x}+\frac{\partial v}{\partial y}+\frac{\partial w}{\partial z}=0 \\
& \frac{\partial u}{\partial t}+u \frac{\partial u}{\partial x}+v \frac{\partial u}{\partial y}+w \frac{\partial u}{\partial z}=g_{x}-\frac{1}{\rho} \frac{\partial p}{\partial x}+\frac{\mu}{\rho}\left(\frac{\partial^{2} u}{\partial x^{2}}+\frac{\partial^{2} u}{\partial y^{2}}+\frac{\partial^{2} u}{\partial z^{2}}\right) \\
& \frac{\partial v}{\partial t}+u \frac{\partial v}{\partial x}+v \frac{\partial v}{\partial y}+w \frac{\partial v}{\partial z}=g_{y}-\frac{1}{\rho} \frac{\partial p}{\partial x}+\frac{\mu}{\rho}\left(\frac{\partial^{2} v}{\partial x^{2}}+\frac{\partial^{2} v}{\partial y^{2}}+\frac{\partial^{2} v}{\partial z^{2}}\right) \\
& \frac{\partial w}{\partial t}+u \frac{\partial w}{\partial x}+v \frac{\partial w}{\partial y}+w \frac{\partial w}{\partial z}=g_{z}-\frac{1}{\rho} \frac{\partial p}{\partial x}+\frac{\mu}{\rho}\left(\frac{\partial^{2} w}{\partial x^{2}}+\frac{\partial^{2} w}{\partial y^{2}}+\frac{\partial^{2} w}{\partial z^{2}}\right)
\end{aligned}
$$

In the equations: $\mathrm{u}, \mathrm{v}, \mathrm{w}$ respectively are velocity vectors in three directions, $\mathrm{p}$, $\rho, \mu$ respectively are pressure, density, dynamic viscosity. 


\section{The Finite Element Model of Level Control Valve of Water Storage Tank}

The level control valve of water storage tank of this paper takes a structure of angle, twostage control including plunger structure of the first stage, throttle orifice plate of the second stage. The valve core range is $67.6 \mathrm{~mm}$.

\subsection{Geometric Model of Flow Channel}

This paper establishes models of flow channel of level control valve of water storage tank in various stages of the open process $(20 \%, 40 \%, 60 \%, 80 \%, 100 \%)$ according to the displacement of the spool using 3D entities modeling software Solidworks. Considering the influence of the valve before and after pipeline on the internal flow field, equip a pipe at the valve inlet and the valve outlet, about 500mm [5]. Figure 1 is the geometric model of flow channel when the valve fully open.

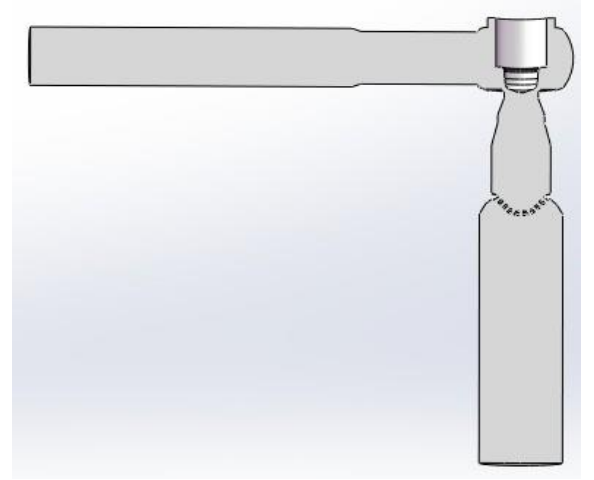

Figure 1. Geometric Model of Flow Channel when the Valve Fully Open

\subsection{Mesh Model of Flow Channel}

The mesh model is generated by the software of ICEM CFD which is CFX Preprocessor. The mesh generation takes unstructured meshes because of the complexity of shape of valve body cavity and flow state of fluid. When set control parameters of grid cell, rational choice of tetrahedron, hexahedron, and the side length and scale of prism should be made. Set a multilayer wall grid. The number of grid is controlled at around 1 million which ensure the calculation accuracy with a appropriate solving speed. Figure 2 is drawing of partial enlargement of mesh model.

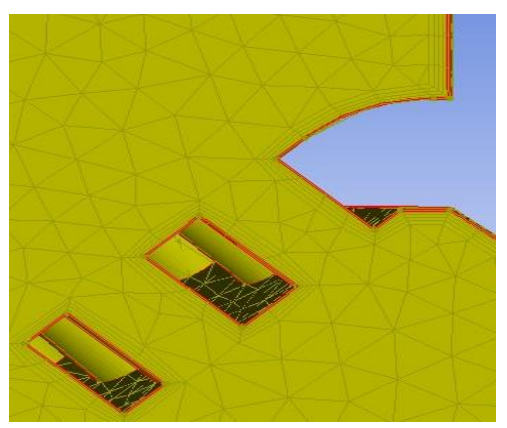

Figure 2. Grawing Enlarged of Mesh Model 


\section{Results of Simulation and Analysis}

The discharge coefficient $K_{v}$ of regulating valve show the flow when the fluid flow through the valve generating unit pressure loss, which reflects the flow capacity of regulating valve. Flow resistance coefficient $\zeta$ show the change of the amount of resistance loss when the valve produce certain flow. The discharge coefficient and flow resistance coefficient are the important performance parameters of level control valve of water storage tank, which depend on valve size, structure and shape of inner chamber, and has nothing to do with medium temperature and flow[6]. In order to make discharge coefficient and flow resistance coefficient more accurate and true, get the curve of ideal flow characteristic and analyze the ideal flow characteristic, keep the valve pressure difference fixed in the simulation analysis. Analyze flow situation of the internal fluid according to the visual graphics of the internal flow field.

\subsection{Solver Parameters and Boundary Conditions}

The fluid medium is water. The reference pressure of fluid domain model is $1 \mathrm{~atm}$, without heat transfer, set turbulence model as $\mathrm{k}$-Epsilon, no burning, no convection, keep valve pressure difference before and after as $1 \mathrm{MPa}$, set the fluid inlet as inlet boundary condition, subsonic flow, static pressure $1 \mathrm{MPa}$. Set the fluid outlet as outlet boundary condition, subsonic flow, static pressure 0MPa.Set the symmetry plane of fluid as a boundary condition of symmetry. Set the tape of wall boundary as no-slip grid. In order to improve the precision, set convection format as the model of upwind, time step as 10,000 steps, convergence precision as 1e-06. The rest of the options keep accordance with the default settings of CFX.

\subsection{Flow Pressure and Flow Lines of Speed}

CFX-Post can display and output the results of simulation and analysis in a variety of ways (pressure contours, velocity vector diagram etc.). Pressure contours, velocity streamline diagrams of the valve in various stages of the open process are shown from Figure 3 to Figure 5 .

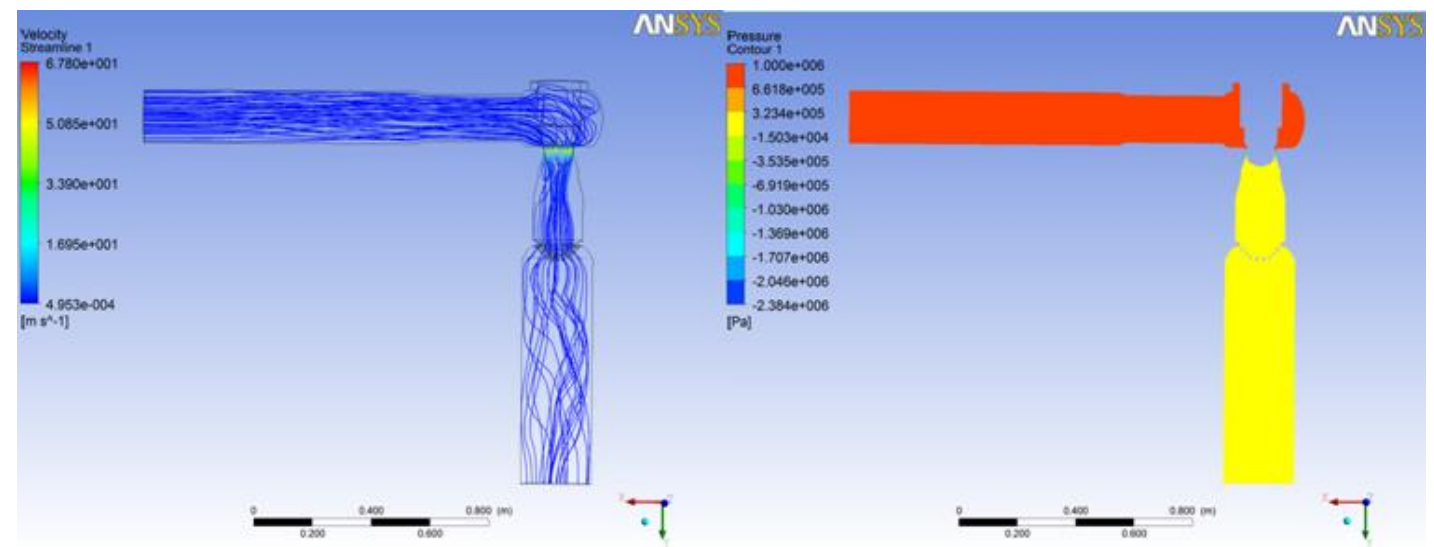

Figure 3. Velocity Streamline Diagram and Pressure Contour When Valve Open $20 \%$ 


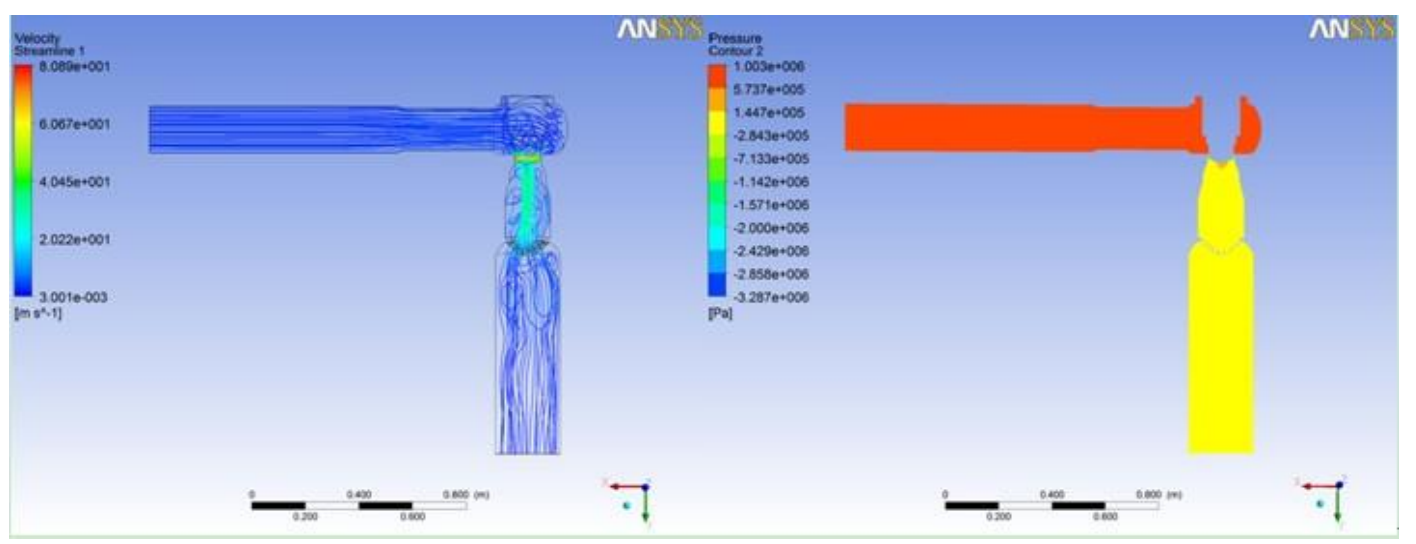

Figure 4. Velocity Streamline Diagram and Pressure Contour When Valve Open $60 \%$

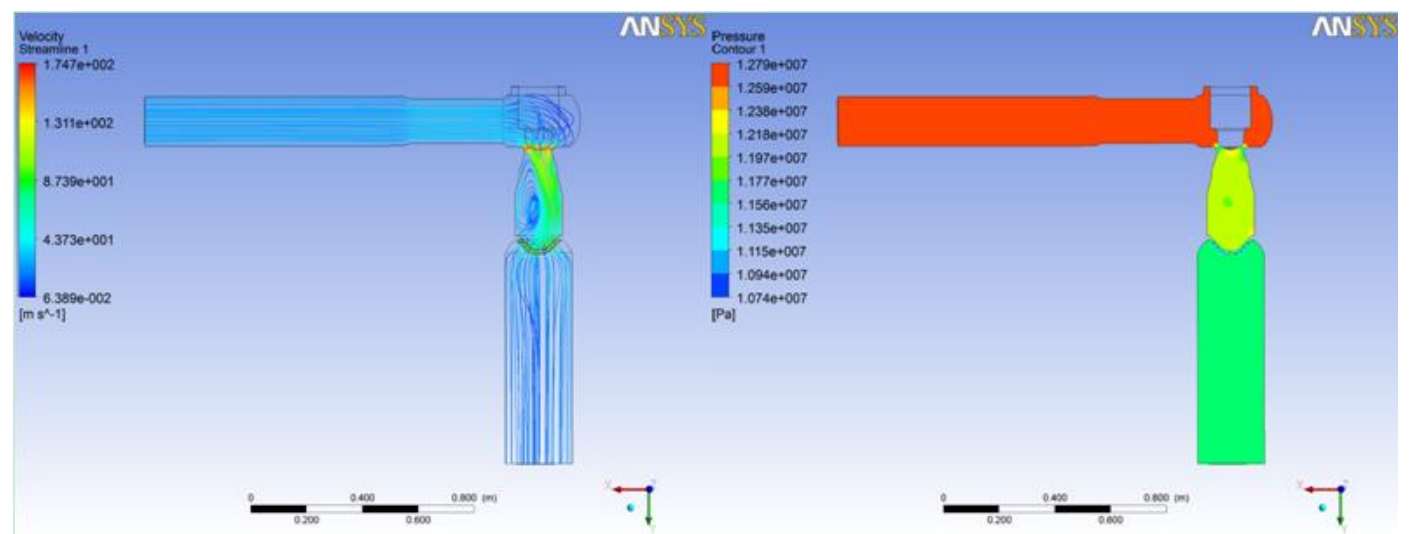

Figure 5. Velocity Streamline Diagram and Pressure Contour when Valve Open Fully

It can be seen from pressure contours, velocity streamline diagrams of the valve in various stages of the open process :Velocity and pressure valves in the valve inlet and outlet pipeline relatively distributes uniformly, which means that computational domain taken could make water flow fully; In the open place of spool, the flow area decreases rapidly and pressure of fluid declines rapidly, however, speed rise; Early in the open process, fluid velocity in the open place of spool rising gradually reaches its maximum value; When valve opening is larger, speed-increase area is moving forward, speed in the open place of spool declines, however, speed around the throttle orifice plate rise. The flow of water can be seen easily from the velocity streamline diagrams: There have eddies appearing in the valve inlet and outlet pipeline; With the valve opening enlarge, eddies in the valve inlet and outlet pipeline disappears, however, eddies appears inside balanced cavity; When the valve nearly open fully, eddies inside balanced cavity still exists, and eddies appears between In the open place of spool and throttle orifice plate.

\subsection{Discharge Coefficient}

For three-dimensional incompressible viscous fluid:

$$
\zeta=\frac{2 \times \Delta p}{\rho \times u^{2}}
$$


In the formula : $\Delta p$-pressure drop of valve

$\zeta$ - The flow resistance coefficient of valve, dimensionless

$\rho$-fluid density

$u$-flow velocity of inlet pipe

$K_{v}=5.09 \times \sqrt{\frac{1}{\zeta}} \times A$

In the formula : $K_{v}$-discharge coefficient, the unit is $\mathrm{t} / \mathrm{h}$

$A$ _cross-sectional area of Inlet pipe, the unit is $\mathrm{cm}^{2}$

$\Delta p$ is $1 \mathrm{MPa}, \rho$ is the density of water $997 \mathrm{~kg} / \mathrm{m} 3$, the post-processor of ANSYS CFX can show inlet pipe flow velocity $u$ in various stages of the open process. Calculate the flow resistance coefficient and discharge coefficient according to formula 1 and formula 2 . Inlet flow velocity, flow resistance coefficient, simulation values of the discharge coefficient, theoretical values and experimental values of the discharge coefficient given by the factory, and the error between simulation values, theoretical values and experimental values of the discharge coefficient. According to Table 1, the error is less than 5\%, which show the reliability of the simulation results.

Table 1. Kv and Error in Various Stages of the Open Process

\begin{tabular}{lccllllll}
\hline $\begin{array}{l}\text { The } \\
\text { relative } \\
\text { opening }\end{array}$ & $\begin{array}{c}\text { displacement } \\
\text { of valve core } \\
(\mathrm{mm})\end{array}$ & $\begin{array}{l}\text { Inlet } \\
\text { flow } \\
\text { velocity } \\
(\mathrm{m} / \mathrm{s})\end{array}$ & $\begin{array}{l}\text { Flow } \\
\text { resistance } \\
\text { coefficient } \\
\zeta\end{array}$ & $\begin{array}{l}\text { Simulation } \\
\text { value of } \\
\mathrm{Kv}(\mathrm{t} / \mathrm{h})\end{array}$ & $\begin{array}{c}\text { theoretical } \\
\text { value of } \\
\mathrm{Kv}(\mathrm{t} / \mathrm{h} \\
)\end{array}$ & $\begin{array}{c}\text { trial } \\
\text { value } \\
\text { of Kv } \\
(\mathrm{t} / \mathrm{h})\end{array}$ & $\begin{array}{c}\text { error o f Kv } \\
\text { (Simulation } \\
\text { and theory }\end{array}$ & $\begin{array}{l}\text { error of } \\
\mathrm{Kv} \\
\text { Simulation } \\
\text { and test })\end{array}$ \\
\hline $20 \%$ & 13.52 & 0.5868 & 5762.76 & 17.72 & 17.21 & 18.08 & 2.88 & 1.99 \\
$40 \%$ & 27.04 & 1.3751 & 1053.36 & 38.99 & 38.10 & 39.94 & 2.28 & 2.38 \\
$60 \%$ & 40.56 & 2.7620 & 262.95 & 78.49 & 77.27 & 79.83 & 1.55 & 1.68 \\
$80 \%$ & 54.08 & 5.1108 & 76.80 & 144.45 & 141.95 & 147.95 & 1.73 & 2.37 \\
$100 \%$ & 67.60 & 7.3759 & 36.83 & 203.43 & 199.36 & 208.38 & 2.00 & 2.38 \\
\hline
\end{tabular}

\subsection{The Curve of Ideal Flow Characteristic}

Fluid characteristics of control valve refers to the relationship between relative flow and relative opening when fluid media flow through the valve [7]. The post processor of ANSYS CFX could display the outlet mass flow in various stages of the open process. According to the dates of outlet mass flow, fit the flow characteristic curve. Table 2 shows the outlet mass flow and relative flow of level control valve of water storage tank in various stages of the open process. Figure 6 shows the flow characteristic curve fitted by the mathematical software MATLAB based on dates of outlet mass flow. 


\section{Table 2. The Outlet Mass Flow and Relative Flow in Various Stages of the Open} Process

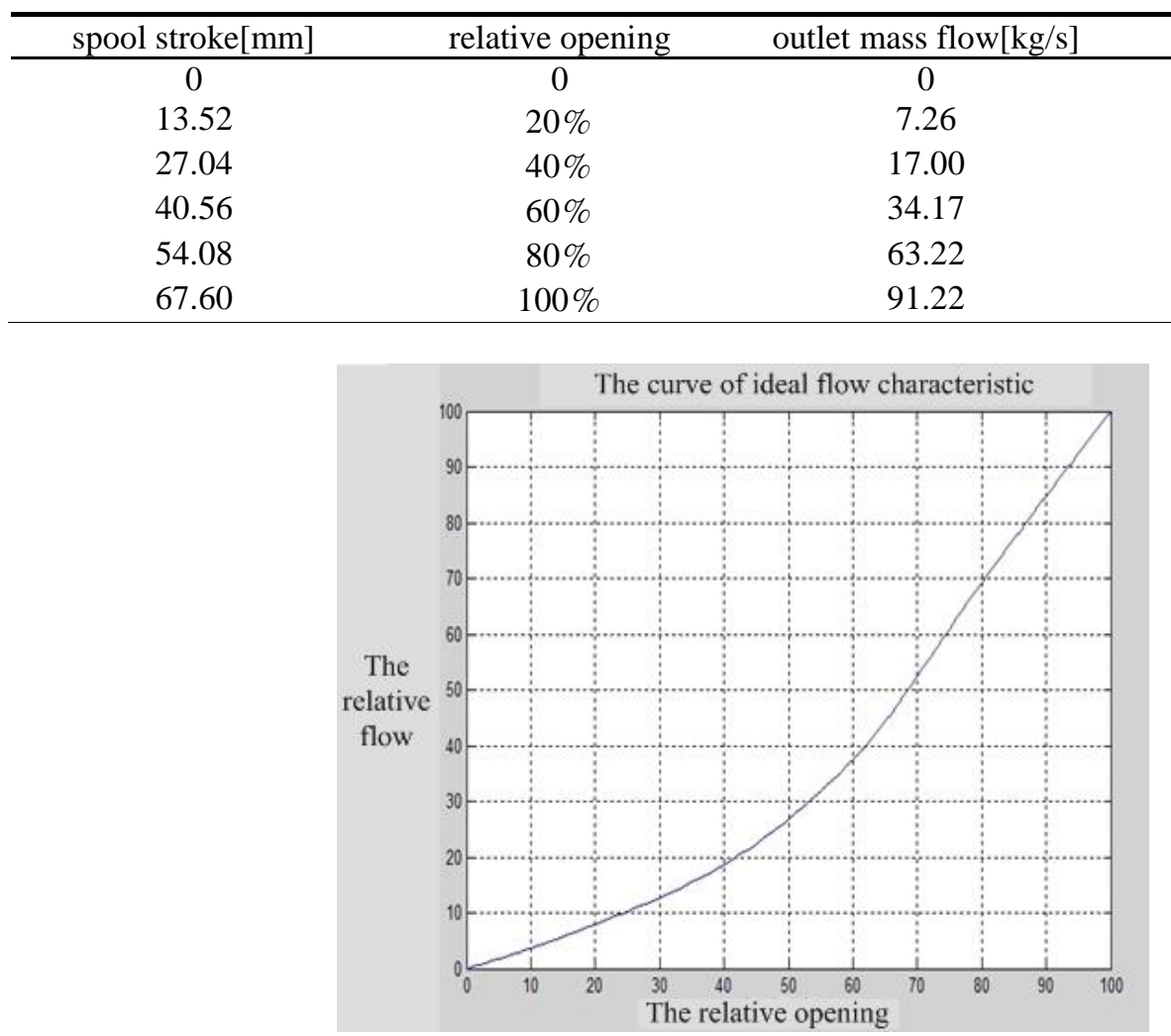

Figure 6. The Curve of Ideal Flow Characteristic

It can be seen from the flow characteristic curve that flow characteristic of the level control valve of water storage tank is closed to the type of equal percentage complying with design of the valve drawings, which show the simulation results are accurate and reliable. When the valve opening is small, the flow is small and the change of flow is small, when the valve opening is large, the flow is large and the change of flow is large, that mean the valve has the same regulation accuracy in various stages of the open process.

\section{Summary}

This paper analyses the significance of level control valve of water storage tank for the safe operation of the ultra-supercritical boilers. Establish flow channel model and mesh models of level control valve of water storage tank in various stages of the open process, and set reasonable solution parameters and boundary conditions. Get pressure contours, velocity streamline diagrams and outlet mass flow of level control valve of water storage tank in various stages of the open process by analysis and calculations using computational fluid dynamics software ANSYS CFX. Get discharge coefficient and flow resistance coefficient. Make detailed analysis of the flow field of level control valve of water storage tank, and fit the flow characteristic curve of level control valve of water storage tank. Provide a theoretical basis for the structural design of level control valve of water storage tank. 
International Journal of Control and Automation

Vol.7, No.10 (2014)

\section{Acknowledgements}

This paper is supported by Enterprise Cooperation Project (2012HVCHT048), International Cooperation Special Project (2012DFA60350).

\section{References}

[1] W. G. Shen and W. J. Yu, "J. Science and Technology Information", vol. 17, (2013).

[2] B. Peng and L. Zhou, "J. Guangxi Electric Power", vol. 2, (2009).

[3] P. Barman, "J. SAE 2002 World Congress", vol. 53, (2010).

[4] J. H. Park, K. H. Kim, C. H. Yeo and H. K. Kim, "J. Plasma Science", vol. 1, no. 42, (2014).

[5] Y. C. Pan, Y. Wang and Y. D. Xie, "J. Machine Tool \& Hydraulics", vol. 1, no. 39, (2011).

[6] X. G. Pan, C. L. Wang, Q. Xia, H. Liu, D. L. Tian and D. L. Qiu, "J. Chinese Hydraulics and Pneumatics", vol. 7, (2013).

[7] P. Barman, "J. SAE 2002 World Congress", vol. 53, (2010). 